\title{
HIGHLIGHTS
}

\section{Graves ophthalmopathy: is oxidative stress the cause?}

Oxidative stress is involved in the pathogenesis of Graves ophthalmopathy (GO) and is not merely a consequence of the disease, suggest the authors of a pilot study from Turkey.

Akarsu and co-investigators evaluated the relationship between GO and levels of two oxidative stress markers-glutathione (an antioxidant) and malondialdehyde (a reactive species that indicates oxidative stress). Furthermore, they

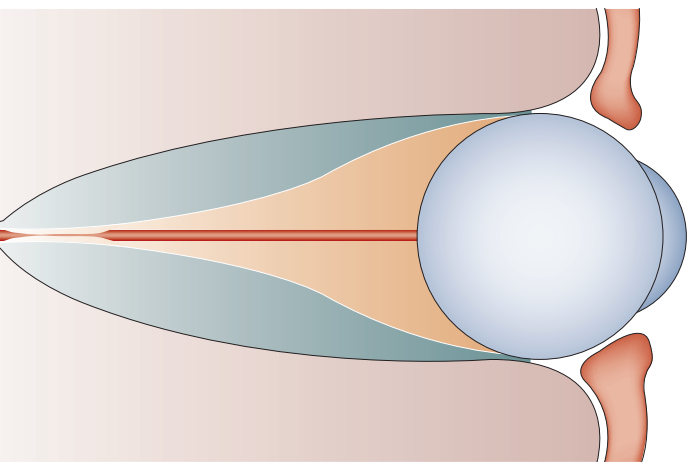

assessed the effects of oral or intravenous methylpredisolone treatment on levels of these markers and on the clinical activity of GO.

Four groups were assessed at three time points (0, 4 and 24 weeks): euthyroid patients with GO treated with either intravenous methypredisolone $(n=18)$ or oral methylpredisolone $(n=15)$ for the first 12 weeks, 20 euthyroid patients with Graves disease but without ophthalmopathy and 15 healthy individuals. Only patients with stable euthyroidism after antithyroid treatment were enrolled in the study to exclude the possible influence of increased levels of thyroid hormones.

At baseline, oxidative stress was indicated in the two groups with GO by higher serum levels of malondialdehyde and lower levels of glutathione than detected in the other two groups. Interestingly, the euthyroid Graves patients without ophthalmopathy had similar levels of these two markers as healthy individuals, which might indicate that oxidative stress had normalized in these patients. Clinical improvement in GO occurred in both groups treated with methylpredisolone, but seemed more rapid in the intravenous than the oral treatment group.

The researchers suggest that malondialdehyde might be a useful marker of GO disease activity, as changes in its levels closely correlated with clinical improvement that occurred over the 24-week study period; by contrast, glutathione levels had not normalized by 24 weeks in the two GO groups.

\section{Carol Wilson}

Original article Akarsu, E. et al. Effects of pulse methylprednisolone and oral methylprednisolone treatments on serum levels of oxidative stress markers in Graves' ophthalmopathy. Clin. Endocrinol. (Oxf.) 74, 118-124 (2010) 